\title{
vuv photoionization of uv-laser-tailored Ni-like $\mathrm{Cu} 3 d^{9}$ atoms
}

\author{
A. Verweyen, ${ }^{1, *}$ A. von dem Borne,${ }^{1}$ P. Glatzel,${ }^{1, \dagger} \mathrm{Ph}$. Wernet, ${ }^{1}$ B. Sonntag ${ }^{1}$ K. Godehusen, ${ }^{2}$ \\ Ch. Gerth, ${ }^{2}$ and P. Zimmermann ${ }^{2}$ \\ ${ }^{1}$ Universität Hamburg, II. Institut für Experimentalphysik, Luruper Chaussee 149, D-22761 Hamburg, Germany \\ ${ }^{2}$ Technische Universität Berlin, Institut für Atomare and Analytische Physik, Hardenbergstraße 36, D-10263 Berlin, Germany
}

(Received 26 February 1999)

\begin{abstract}
$\mathrm{Cu}$ atoms have been prepared in the Ni-like $\mathrm{Cu} 3 d^{9} 4 s^{2}$ state by uv-laser pumping. In contrast to the ground-state atoms, the $\mathrm{Cu} 3 d^{9} 4 s^{2}$ atoms exhibit a prominent asymmetric resonance in the $3 d^{-1}$ partial cross section at the $3 p$ threshold. A close similarity to the corresponding resonance in the spectrum of Ni $3 d^{9} 4 s$ atoms is demonstrated. [S1050-2947(99)50808-1]
\end{abstract}

PACS number(s): 32.80.Fb, 32.80.Dz

Core-level photoelectron spectroscopy for the elementspecific investigation of solids has been greatly enhanced by combining it with electron spin-polarization analysis and the measurement of magnetic dichroism; the ferromagnetic $3 d$ transition metals $\mathrm{Fe}, \mathrm{Co}, \mathrm{Ni}$; and the neighboring elements $\mathrm{Cr}$ and $\mathrm{Mn}$, bound on surfaces, in ultrathin films and multilayers, have attracted particular interest ([1-7] and references therein). Corresponding measurements on free oriented metal atoms can yield detailed information on the importance of intra-atomic effects. The extension of the pioneering study on the dichroism of free laser-oriented $\mathrm{Cr}$ atoms [8] to $\mathrm{Mn}-\mathrm{Ni}$ atoms, however, is hampered by experimental difficulties. The strong optical transitions for the laser pumping process lie in the uv below $380 \mathrm{~nm}$. In an approach to this wavelength regime we have successfully implemented a frequency-doubling scheme.

Atomic $\mathrm{Ni}$ is probably the most interesting but also the most difficult sample in the Mn-Ni series with respect to the preparation of a laser-oriented atomic beam. At a temperature of $1500{ }^{\circ} \mathrm{C}$, which is required for a vapor pressure of $10^{-2} \mathrm{~mm} \mathrm{Hg}$ [9], Ni becomes an aggressive melt and must be contained in ceramic, since it alloys with refractory metals. Furthermore, at these elevated temperatures $60 \%$ of the $\mathrm{Ni}$ atoms are thermally excited, thereby preventing their participation in the optical pumping process. Even worse, half of the $\mathrm{Ni}$ atoms are distributed over the two initial configurations $3 d^{8} 4 s^{2}$ and $3 d^{9} 4 s$; this enormously complicates the interpretation of the photoelectron spectrum. In order to bypass these difficulties, we prepared a beam of free $\mathrm{Cu}$ atoms in an open- $3 d$-shell "Ni-like" state. The metastable $\mathrm{Cu}^{*} 3 d^{9} 4 s^{2}$ atoms were investigated with respect to their suitability as a model system for the $3 p$ photoionization of free $\mathrm{Ni} 3 d^{9} 4 s$ atoms.

In the experiment a collimated beam of $\mathrm{Cu}$ atoms was produced with a high-temperature metal atom furnace [10] by resistively heating a $\mathrm{Ta}$ crucible containing the $\mathrm{Cu}$ metal to approximately $1250{ }^{\circ} \mathrm{C}$. The atomic beam was intersected by uv-laser radiation and vuv synchrotron radiation propagating in opposite directions. The laser was used to prepare

\footnotetext{
*Present address: HASYLAB at DESY, Notkestraße 85, D-22603 Hamburg, Germany.

${ }^{\dagger}$ Present address: Lawrence Berkeley National Laboratory, Berkeley, CA.
}

the $\mathrm{Cu}$ atoms in an excited metastable $3 d^{9}$ state, as will be explained below. The $3 p$ electrons of the metastable $\mathrm{Cu}^{*}$ atoms were then excited with synchrotron radiation from the U1 TGM-6 high-flux undulator station at the electron storage ring BESSY I in Berlin. Photoelectrons emitted at angles close to the magic angle $\Theta_{\mathrm{CMA}}=54.7^{\circ}$ relative to the polarization axis of the synchrotron radiation were detected by a $180^{\circ}$ cylindrical mirror analyzer (CMA) with an energy resolution of $E_{\text {pass }} / \Delta E_{\text {pass }}=125$. All electron spectra in this work have been corrected for the analyzer transmission and normalized to the incoming photon flux. The calibration of the photon energy was established by measuring the $\mathrm{Xe} 4 d_{5 / 2}$ $\rightarrow n p$ and $\mathrm{Kr} 3 d_{3 / 2} \rightarrow n p$ resonance positions [11]. More details on the experimental setup can be found in Ref. [12].

The preparation of the metastable $\mathrm{Cu}^{*}$ atoms is indicated in Fig. 1. An $\mathrm{Ar}^{+}$-laser-pumped single-mode $\mathrm{cw}$ ring dye laser operated with DCM provided $800 \mathrm{~mW}$ at $650 \mathrm{~nm}$ and approximately $5-10 \%$ in the second harmonic, generated in an external ring resonator. Note that the hyperfine structure ([13], and references therein) introduced by the nuclear spin $I=3 / 2$ is not shown in Fig. 1. In the experiment the strongest $4 s \rightarrow 4 p_{3 / 2}$ transition $F=2 \rightarrow F^{\prime}=3$ of the more abundant isotope ${ }^{63} \mathrm{Cu}(69 \%)$ was excited with a laser bandpass of 1

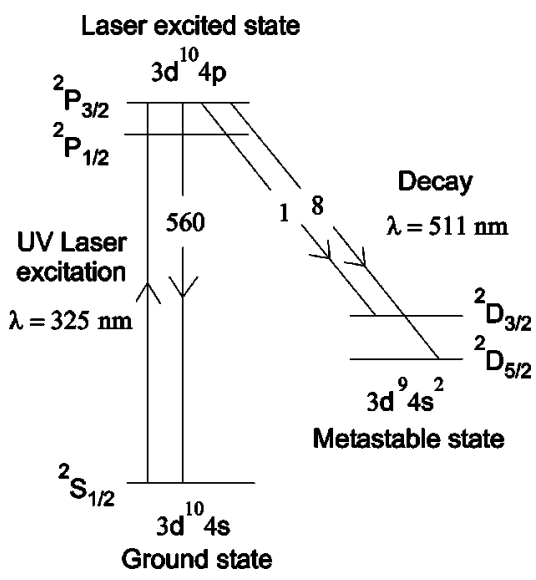

FIG. 1. Illustration of the uv-laser tailoring process in an energy-level scheme. The five lowest fine-structure levels of $\mathrm{Cu}$ I are shown. A small fraction of the laser-excited $3 d^{10} 4 p^{2} P_{3 / 2}$ atoms decays into the metastable state $3 d^{9} 4 s^{2}$ by emitting green fluorescence light. The numbers on the arrows give the relative transition probabilities [15]. 


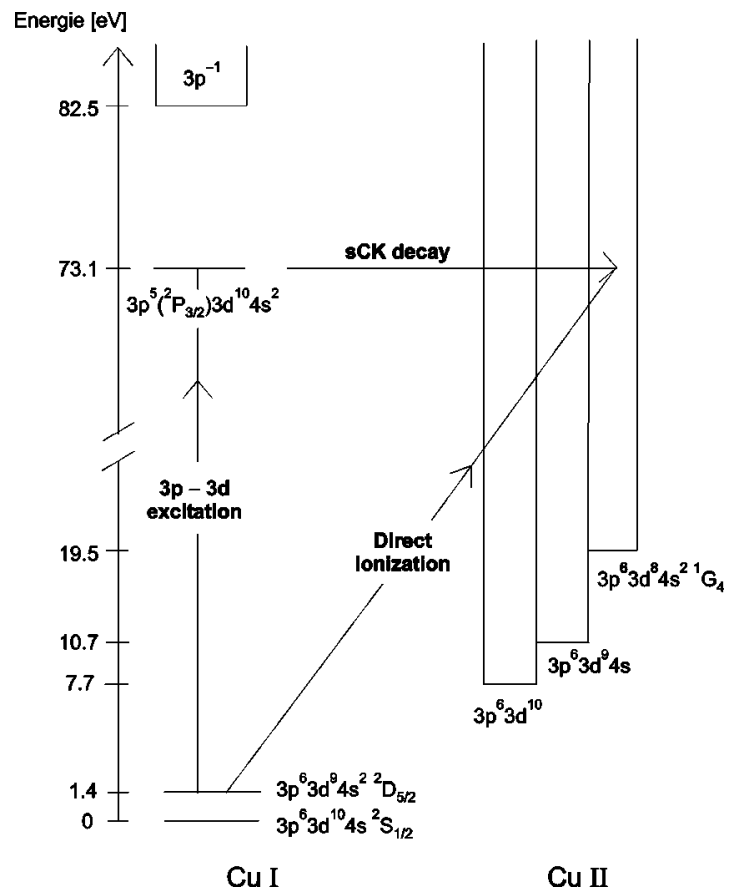

FIG. 2. vuv energy level scheme of $\mathrm{Cu}$ atoms. Interference between direct $3 d$ photoionization of metastable $\mathrm{Cu}$ atoms and $3 p$ excitation followed by autoionization is indicated by arrows. The vuv energies are based on high-resolution absorption spectroscopy [16] and photoelectron spectroscopy [17].

$\mathrm{MHz}$ at $325 \mathrm{~nm}$. The laser-excited $3 d^{10} 4 p$ state has a small admixture of $3 d^{9} 4 s 4 p$ [14] that can decay to the metastable $3 d^{9} 4 s^{2}$ state by a one-electron transition; the corresponding probability (relative to a decay back into the ground state) amounts to $\sim 1 \%$ [15]. From the ${ }^{2} P_{3 / 2}$ laser-excited state, the ${ }^{2} D_{5 / 2}$ metastable state is populated much more effectively than the ${ }^{2} D_{3 / 2}$ metastable state (ratio 8:1 [15]); approximately $5-10 \%$ of the atoms could be prepared in the metastable $\mathrm{Cu}^{*}$ state.

The inner-shell excitation and decay processes are illustrated in the simplified energy-level diagram of Fig. 2. It displays the excitation of the $3 p^{5}\left({ }^{2} P_{3 / 2}\right) 3 d^{10} 4 s^{2}$ resonance state at $73.1 \mathrm{eV}$ [16] from the metastable state $3 p^{6} 3 d^{9}\left({ }^{2} D_{5 / 2}\right) 4 s^{2}$. Since for the $3 p^{5} 3 d^{10} 4 s^{2}$ states the $3 d$ shell is filled, there is only the $3 p$ core-hole spin-orbit splitting of $2.3 \mathrm{eV}$ [17]. The first $3 p^{-1}$ ionization threshold $3 p^{5}\left({ }^{2} P_{3 / 2}\right) 3 d^{10} 4 s$ at $82.5 \mathrm{eV}$ [17] is also depicted. The decay of the $3 p^{5}\left({ }^{2} P_{3 / 2}\right) 3 d^{10} 4 s^{2}$ resonance by a super CosterKronig (sCK)-type autoionization transition into the $3 p^{6} 3 d^{8}\left({ }^{1} G_{4}\right) 4 s^{2} \varepsilon f$ continuum is marked by an arrow in Fig. 2. Interference between this indirect ionization channel and the direct $3 d$ ionization is expected to give rise to an asymmetric Fano-type resonance profile.

The photoelectron spectrum of atomic $\mathrm{Cu}$ in the valence energy region, taken at a photon energy of $\hbar \omega=71.9 \mathrm{eV}$, is given by the dashed line connecting the open circles in Fig. 3 . The photon energy had been tuned to the $3 p_{3 / 2} \rightarrow 3 d$ resonance maximum of the metastable $\mathrm{Cu}^{*}$ atoms (see Fig. 2) in order to enhance their weak $3 d^{-1}$ photoelectron signal. The dominating feature in Fig. 3 is the $3 d^{-1}$ main photoelectron line of $\mathrm{Cu}$ atoms in the ground state at a binding energy of

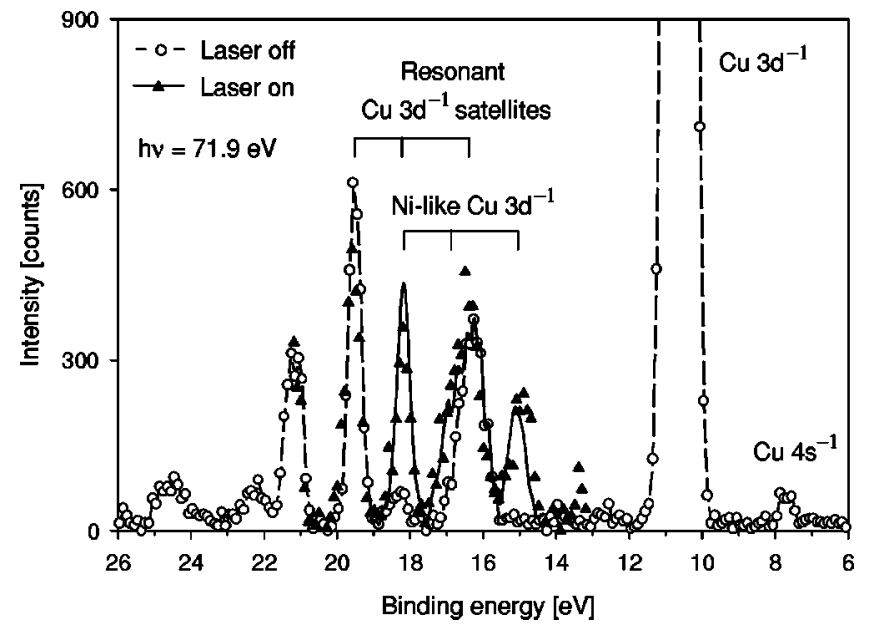

FIG. 3. Photoelectron spectrum of $\mathrm{Cu}$ atoms in the valence energy region, recorded at a photon energy of $\hbar \omega=71.9 \mathrm{eV}$. The spectrum was taken with the laser radiation tuned to the $4 s \rightarrow 4 p$ resonance $\left(-\boldsymbol{\Delta}_{-}\right)$and without laser radiation (-- $\left.\bigcirc--\right)$.

$10.7 \mathrm{eV}$, stemming from the $3 d^{10} 4 s \rightarrow 3 d^{9} 4 s \varepsilon l$ photoionization process. In comparison, the $4 s^{-1}$ main line below $8 \mathrm{eV}$ is hardly visible.

In the energy region from 12 to $20 \mathrm{eV}$, most of the photoelectron lines are due to multiplet-split $3 d^{8} 4 s^{2}$ final ionic states. Since the ground state and the metastable state have the same parity, the same states can be accessed from them in a dipole transition. The lines observed at binding energies of $16.5,18.3$, and $19.6 \mathrm{eV}$ are resonantly enhanced by the $3 p^{6} 3 d^{10} 4 s^{2} S_{1 / 2} \rightarrow 3 p^{5}\left({ }^{2} P_{3 / 2}\right) 3 d^{10} 4 s^{2}$ $\rightarrow 3 p^{6} 3 d^{8}\left({ }^{3} F,{ }^{1} D,{ }^{1} G\right) 4 s^{2} \varepsilon l$ excitation and decay process starting from the $\mathrm{Cu}$ ground state $[18,19] .{ }^{1}$ In the region of 16.0-16.9 eV the $3 d^{9} 4 p$ final ionic states also contribute; they are not affected by the $3 p$ resonances.

The second set of data shown in Fig. 3 has been taken with laser radiation on (solid triangles approximated by the solid line). In the energy region from 12 to $20 \mathrm{eV}$, the photoelectron intensity is strongly modified by the laser radiation. The photoelectron lines from the metastable $\mathrm{Cu}^{*}$ atoms $^{2}$ are shifted by $1.4 \mathrm{eV}$ to lower binding energies, in comparison with the corresponding lines of ground-state $\mathrm{Cu}$ atoms. Therefore the $\mathrm{Cu}^{*}$ photoelectron lines at 15.1, 16.9, and $18.2 \mathrm{eV}$ in the spectrum taken with the laser radiation on (Fig. 3) are assigned to the same final ionic $3 d^{8} 4 s^{2}$ states $\left({ }^{3} F,{ }^{1} D,{ }^{1} G\right)$ discussed above. Starting from the $3 d^{9} 4 s^{2}$ metastable state instead of the ground state, the $3 d^{8} 4 s^{2}$ final ionic states can also be reached simply by the ionization of a $3 d$ electron.

The $3 d^{-1}$ partial cross section of the Ni-like $\mathrm{Cu}$ atoms was measured by recording the intensity variation of the pho-

\footnotetext{
${ }^{1}$ Although the maximum of the $3 p^{6} 3 d^{10} 4 s \rightarrow 3 p^{5}\left({ }^{2} P_{3 / 2}\right) 3 d^{10} 4 s^{2}$ resonance lies at $73.1 \mathrm{eV}$ [16], it is already excited $1.2 \mathrm{eV}$ lower due to the large lifetime width of $\Gamma_{3 p}=1.8 \mathrm{eV}$ (full width at half maximum) $[20,21]$.

${ }^{2}$ The $3 d^{10} 4 p \rightarrow 3 d^{9} 4 p \varepsilon l$ lines from the short-lived laser-excited atoms expected at binding energies of $12.2-13.1 \mathrm{eV}$ [14] are not included in Fig. 3.
} 
toelectron line at $E_{B}=18.2 \mathrm{eV}$ (see Fig. 3) while scanning the photon energy (CIS spectrum). In order to correct for the contribution of the ground-state atoms, two spectra were taken simultaneously by switching the laser beam on and off for every datum point using a computer-controlled shutter. The difference of these two spectra, which directly gives the $3 d^{9} 4 s^{2} \rightarrow 3 d^{8}\left({ }^{1} G\right) 4 s^{2} \varepsilon l$ partial photoionization cross section, is depicted in Fig. 4 (solid points with error bars). The spectrum displays the broad $3 p^{6} 3 d^{9} 4 s^{2}$ $\rightarrow 3 p^{5}\left({ }^{2} P_{3 / 2}\right) 3 d^{10} 4 s^{2}$ resonance ${ }^{3}$ with a pronounced asymmetry corresponding to a positive Fano $q$ parameter. Similar $3 p \rightarrow 3 d$ resonances are characteristic of the vuv spectra of the open $3 d$-shell transition-metal atoms; they comprise a considerable fraction of the oscillator strength for transitions from the $3 p$ subshell $[22,23]$. The spin-orbit partner state $3 p^{5}\left({ }^{2} P_{1 / 2}\right) 3 d^{10} 4 s^{2}$ cannot be excited from the metastable state $3 d^{9}\left({ }^{2} D_{5 / 2}\right) 4 s^{2}$ predominantly populated in the lasertailoring process; excitation from the weakly populated $3 d^{9}\left({ }^{2} D_{3 / 2}\right) 4 s^{2}$ metastable state is not visible in the spectrum of Fig. 4.

For comparison, the $3 d^{-1}$ partial photoionization cross section of ground-state $\mathrm{Cu} 3 p^{6} 3 d^{10} 4 s$ atoms, measured at $E_{B}=10.7 \mathrm{eV}$ (see Fig. 3), is included in Fig. 4 (open circles approximated by the dashed line). It was scaled by a factor of 0.075 (as a result of a fitting procedure; see below), which reflects the small fraction of atoms in the metastable state. The ground-state spectrum, being hardly affected by $3 p$ excitations, differs markedly from the spectrum of the metastable state.

The $3 p_{3 / 2} \rightarrow 3 d$ resonance in the spectrum of Ni-like $\mathrm{Cu}$ atoms can be well approximated by a Fano-type profile (solid line in Fig. 4). The resonance energy of $71.70 \mathrm{eV}$ and the resonance width of $1.84 \mathrm{eV}$ were derived from spectra of ground-state atoms displaying the $3 p^{6} 3 d^{10} 4 s$ $\rightarrow 3 p^{5}\left({ }^{2} P_{3 / 2}\right) 3 d^{10} 4 s^{2}$ resonance with much better statistics. For the fit of the Fano profile a noninteracting continuum [24] was taken into account that was matched to the groundstate $3 d^{-1}$ continuum of Fig. 4; the additional $4 s$ electron should not make a large difference. The approximation resulted in an Fano asymmetry parameter of $q=+2.6 \pm 1$; the considerable uncertainty is due to the statistical scatter of the data.

It is very instructive to compare the partial cross section of the Ni-like $\mathrm{Cu}$ atoms with the analogous cross section $3 p^{6} 3 d^{9} 4 s \rightarrow 3 p^{6} 3 d^{8}\left({ }^{1} G\right) 4 s \varepsilon l$ of excited Ni atoms. The corresponding $\mathrm{Ni}$ spectrum $[25,26]$ is given by the open diamonds in Fig. 4; it displays a strong asymmetric resonance attributed to a $3 p^{6} 3 d^{9} 4 s \rightarrow 3 p^{5}\left({ }^{2} P_{3 / 2}\right) 3 d^{10} 4 s$ excitation. In Fig. 4, the Ni spectrum was shifted by $9 \mathrm{eV}$ to higher energies and the intensity of the Ni resonance was matched to the $\mathrm{Ni}$-like $\mathrm{Cu}$ resonance. The shapes of the $\mathrm{Ni}$ and the Ni-like $\mathrm{Cu}$ resonances are very similar. A detailed inspection reveals that there is possibly a weak contribution from the Ni $3 p_{1 / 2}$ $\rightarrow 3 d$ spin-orbit partner on the high-energy side of the $3 p_{3 / 2} \rightarrow 3 d$ resonance. The corresponding splitting would be

${ }^{3} \mathrm{~A}$ CIS spectrum of the $3 d^{-1}$ electron lines at $E_{B}=16.9 \mathrm{eV}$ (see Fig. 3), belonging to a ${ }^{3} F$ multiplet of the ionic $3 d^{8}$ shell, also displays the $3 p_{3 / 2} \rightarrow 3 d$ resonance.

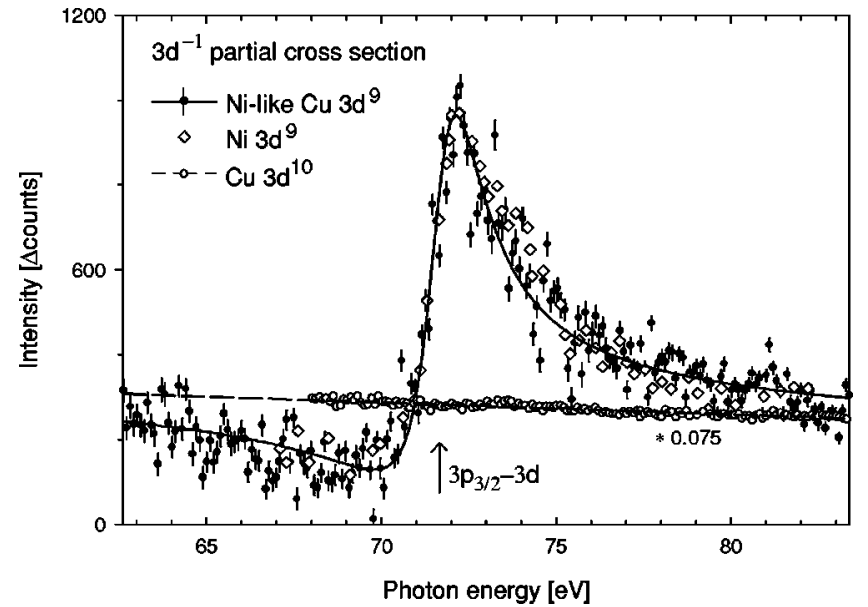

FIG. 4. Experimental $3 d^{-1}$ partial cross sections of Ni-like $\mathrm{Cu}$ $\left(-\bigcirc-, E_{B}=18.2 \mathrm{eV}\right), \mathrm{Ni}(\diamond$, from Ref. [25]) and $\mathrm{Cu}$ atoms $\left(--\bigcirc--, E_{B}=10.7 \mathrm{eV}\right)$ in the region of the $3 p$ inner-shell excitations. The $\mathrm{Cu}$ spectrum was scaled by a factor of 0.075 , the Ni spectrum was shifted by $9 \mathrm{eV}$ to higher binding energies and scaled in order to match the Ni-like $\mathrm{Cu}$ spectrum. The error bars in the Ni-like $\mathrm{Cu}$ spectrum reflect the statistical scatter.

in good agreement with a spin-orbit splitting of $1.8 \mathrm{eV}$ [27]. However, the relative strength of the $3 p_{1 / 2} \rightarrow 3 d$ component is significantly smaller than the statistical weight of $1: 2$, as has been noted earlier $[25,26]$. A transfer of this result to the $\mathrm{Ni}$-like $\mathrm{Cu}$ atoms, additionally taking into account that the $\mathrm{Cu}^{*} 3 p_{12} \rightarrow 3 d$ resonance cannot be excited from the ${ }^{2} D_{5 / 2}$ metastable state, could explain why there is no indication of the spin-orbit partner in the spectrum of metastable $\mathrm{Cu}$.

In conclusion, we have determined the emission of $3 d$ electrons in the photoelectron spectrum of $\mathrm{Cu}^{*}$ atoms that are laser-tailored in a metastable Ni-like $3 d^{9} 4 s^{2}$ state. The $3 d^{-1}$ main line intensity varies strongly when the photon energy is scanned through the $3 p$ thresholds. A characteristic broad and asymmetric resonance shows up in the corresponding CIS spectrum; this resonance, which can be well approximated by a Fano-type profile, is attributed to a transition of a $3 p_{3 / 2}$ electron into the $3 d$ orbital opened in the laser-tailoring process. There is no indication of a contribution from the $3 p_{1 / 2} \rightarrow 3 d$ spin-orbit partner. A detailed comparison showed that the measured CIS spectrum of the Nilike $\mathrm{Cu}$ atoms is very similar to the corresponding spectrum of excited Ni $3 d^{9} 4 s$ atoms. Therefore, $3 p$ photoionization of the metastable $\mathrm{Cu}$ atoms can serve as a model for $3 p$ photoionization of $\mathrm{Ni} 3 d^{9} 4 s$ atoms. In this context it is very interesting that the photoelectron spectra of small, negatively charged $\mathrm{Ni}_{n}^{-}$and $\mathrm{Cu}_{n}^{-}$clusters show marked similarities indicating a strong localization of the $3 d$ orbitals [28]. It would be very interesting to compare the $3 p$ photoionization of metastable $\mathrm{Cu}$ atoms and $\mathrm{Ni}$ atoms bound in small clusters.

In our experiment the small number of metastable atoms precluded photoelectron spectroscopy above the $3 p$ thresholds. In a future experiment, a second laser tuned to the transition between the state excited by the first laser and the metastable state will be used in order to transfer the $\mathrm{Cu}$ atoms more effectively from the ground state to the metastable state. Alternatively, improving the flux of the vuv light 
source, as expected for example for the free-electron laser test facility TESLA at DESY [29], would bring progress.

As we have shown above, in contrast to the $\mathrm{Cu}^{*} 3 d^{9} 4 s^{2}$ atoms, the $3 d^{-1}$ partial cross section of the $\mathrm{Cu} 3 d^{10} 4 s$ ground-state atoms displays only a little resonant structure at the $3 p$ threshold. Therefore resonant photoelectron spectroscopy at the $3 p$ threshold offers a very sensitive test of the $3 d$ shell occupation of $\mathrm{Cu}$ atoms or ions in compounds (see, e.g., [30-33], and references therein).

We thank V. K. Ivanov for stimulating discussions, and I. Schubert and M. Grothe for their help at the beamline. Assistance of the BESSY staff and financial support by the Deutsche Forschungsgemeinschaft are gratefully acknowledged.
[1] P. D. Johnson, Rep. Prog. Phys. 60, 1217 (1997).

[2] M. Liberati, G. Panaccione, F. Sirotti, P. Prieto, and G. Rossi, Phys. Rev. B 59, 4201 (1999).

[3] J. G. Menchero, Phys. Rev. B 57, 1001 (1998).

[4] H. A. Dürr, G. van der Laan, D. Spanke, F. U. Hillebrecht, and N. B. Brookes, J. Electron Spectrosc. Relat. Phenom. 93, 233 (1998).

[5] C. M. Schneider, U. Pracht, W. Kuch, A. Chassé, and J. Kirschner, Phys. Rev. B 54, R15 618 (1996).

[6] J. Stöhr, J. Electron Spectrosc. Relat. Phenom. 75, 253 (1995).

[7] F. M. F. de Groot, J. Electron Spectrosc. Relat. Phenom. 67, 529 (1994).

[8] A. von dem Borne, T. Dohrmann, A. Verweyen, B. Sonntag, K. Godehusen, and P. Zimmermann, Phys. Rev. Lett. 78, 4019 (1997).

[9] K. J. Ross and B. Sonntag, Rev. Sci. Instrum. 66, 4409 (1995).

[10] Th. Dohrmann, dissertation, Universität Hamburg, 1995.

[11] G. C. King, M. Tronc, F. H. Read, and R. C. Bradford, J. Phys. B 10, 2479 (1977).

[12] Th. Dohrmann, A. von dem Borne, A. Verweyen, B. Sonntag, M. Wedowski, K. Godehusen, P. Zimmermann, and V. Dolmatov, J. Phys. B 29, 4641 (1996).

[13] J. Bengtsson, J. Larsson, S. Svanberg, and C.-G. Wahlström, Phys. Rev. A 41, 233 (1990).

[14] J. Sugar and A. Musgrove, J. Phys. Chem. Ref. Data 19, 527 (1990).

[15] K. Fu, M. Jogwich, M. Knebel, and K. Wiesemann, At. Data Nucl. Data Tables 61, 1 (1995).

[16] R. Bruhn, B. Sonntag, and H. W. Wolff, J. Phys. B 12, 203 (1979).

[17] Ch. Gerth, K. Tiedtke, M. Martins, B. Obst, P. Zimmermann,
P. Glatzel, A. Verweyen, Ph. Wernet, and B. Sonntag, J. Phys. B 31, 2539 (1998).

[18] D. Chandesris, C. Guillot, G. Chauvin, J. Lecante, and Y. Petroff, Phys. Rev. Lett. 47, 1273 (1981).

[19] R. Bruhn, E. Schmidt, H. Schröder, and B. Sonntag, J. Phys. B 15, L441 (1982).

[20] L. C. Davis and L. A. Feldkamp, Phys. Rev. A 24, 1862 (1981).

[21] F. Combet Farnoux, Z. Phys. D 2, 337 (1986).

[22] B. Sonntag and P. Zimmermann, Rep. Prog. Phys. 55, 911 (1992).

[23] L. C. Davis, J. Appl. Phys. 59, R25 (1986).

[24] U. Fano and L. Cooper, Rev. Mod. Phys. 40, 441 (1968).

[25] M. Meyer, Th. Prescher, E. von Raven, M. Richter, E. Schmidt, B. Sonntag, and H. Wetzel, Z. Phys. D 2, 347 (1986).

[26] E. Schmidt, H. Schröder, B. Sonntag, and H. E. Wetzel, J. Phys. B 16, 2961 (1983).

[27] R. E. Dietz, E. G. McRae, and J. H. Weaver, Phys. Rev. B 21, 2229 (1980).

[28] G. Ganteför and W. Eberhardt, Phys. Rev. Lett. 76, 4975 (1996).

[29] J. Feldhaus and B. Sonntag, Synchrotron Rad. News 11, 21 (1998).

[30] J. Ghijsen, L. H. Tjeng, H. Eskes, G. A. Sawatzky, and R. L. Johnson, Phys. Rev. B 42, 2268 (1990).

[31] H. Maack, diplomarbeit, Universität Hamburg, 1985.

[32] M. R. Thuler, R. L. Benbow, and Z. Hurych, Phys. Rev. B 26, 669 (1982).

[33] M. Klauda, J. Markl, C. Fink, P. Lunz, G. Saemann-Ischenko, F. Rau, K.-J. Range, R. Seemann, and R. L. Johnson, Phys. Rev. B 48, 1217 (1993). 